\title{
Stenosis and Aneurysm of Coronary Arteries in A Patient with Behcet's Disease
}

\author{
Hammami Sonia ${ }^{1, *}$, Ben Hamda Khaldoun ${ }^{2}$, Mahjoub Sylvia ${ }^{1}$, Maatoug Faouzi ${ }^{2}$, Gamra Habib ${ }^{1}$ and \\ Ben Farhat Mohamed ${ }^{1}$
}

${ }^{1}$ Department of Internal Medicine, ${ }^{2}$ Department of Cardiology, University Hospital “F. Bourguiba” Monastir (Tunisia)

\begin{abstract}
Coronary artery disease is extremely rare in patients with Behçet's disease. We report the case of a patient with a history of Behçet's disease who was admitted in our hospital with instable angina pectoris. The patient's electrocardiogram was normal. Coronary angiography revealed aneurysm of the distal right coronary artery with a tight stenosis of the proximal part of the posterolateral branch. These two conditions were initially treated with immunosuppressive treatment. Three years later coronary angiography showed a total occlusion of the right coronary artery treated with medical therapy. More than fourteen cases of coronary involvement were reported in the literature but the etiopathogeny and the treatment are yet unknown
\end{abstract}

Key Words: Behçet's disease, coronary aneurysm, stenosis.

\section{INTRODUCTION}

Behçet's disease (BD) is known as a chronic relapsing inflammatory process manifesting recurrent oral and genital aphtous ulcerations, skin eruptions, eye inflammation and neurological manifestations. The spectrum of the disease is ever expanding, involvement of joints, gastrointestinal and respiratory systems are also recognised [1]. Cardiovascular involvement is a serious complication, although involvement of coronary arteries is extremely rare and often associated with poor prognosis [2-4]. This case study presents a patient with BD who developed coronary artery aneurysm and stenosis.

\section{CASE REPORT}

A 50-year-old Tunisian man, diagnosed with BD since the age of 29 years, was hospitalized for angina pectoris with a 2 weeks history of anterior chest pain (Canadian Cardiovascular Society class III). The BD diagnoses were based on oral aphta and genital ulcers with a positive pathergy test and repeated episodes of posterior segment inflammation treated by corticosteroids but complicated by complete blindness. The only cardiac risk factor was smoking. Physical examination upon admission revealed a fever of $37.5^{\circ} \mathrm{C}$, pulse rate at 90 beats/ min and blood pressure at $120 / 70 \mathrm{mmHg}$. Heart sounds and bilateral lung area were normal. The patient erythrocyte sedimentation rate was at $30 \mathrm{~mm} / \mathrm{hr}, \mathrm{C}$ reactive protein level at $6 \mathrm{mg} / \mathrm{dl}$. The protein $\mathrm{C}, \mathrm{S}$, homocyteinemia, creatine kinase, serum glutamic-oxaloacetic transaminase, liver and kidney function tests, serum cholesterol and triglycerides were all within the normal limits. Anticardiolipin antibodies and antinuclear factor were negative. An electrocardiogram revealed only sinus tachycardia. Transthoracic echocardiogram revealed no abnormality. On the base

\footnotetext{
*Address correspondence to this author at the Department of Internal Medicine, University Hospital F. Bourguiba, 5019, Monastir, Tunísia; Tel: 216 73 447; 984 Fax: 21673460 737; E-mail: Sonia.Hammami@fmm.rnu.tn
}

of these data, the patient was diagnosed with instable angina. Treatment was initiated with Colchicine, intravenous nitroglycerin, heparine and beta-blocker therapies which lead to a resolution of the symptoms. Six days after hospital admission, the patient underwent coronary angiography, showing one aneurysm $(16 / 8 \mathrm{~mm})$ located on the distal right coronary artery with a tight stenosis located on the proximal part of the posterolateral branch (Fig. 1). Heparine therapy was stopped and the following course of treatment implemented: monthly pulse of intravenous cyclophosphamide, methylprednisolone $1 \mathrm{~g} /$ day for 3 days followed by oral administration of prednisone at a dose of $80 \mathrm{mg} /$ day leading to improvement of all the symptoms. The dose of prednisolone was gradually decreased. Three years later, the patient was referred to cardiac unit with chest pain; coronary angiography showed a total occlusion of the right coronary with athe

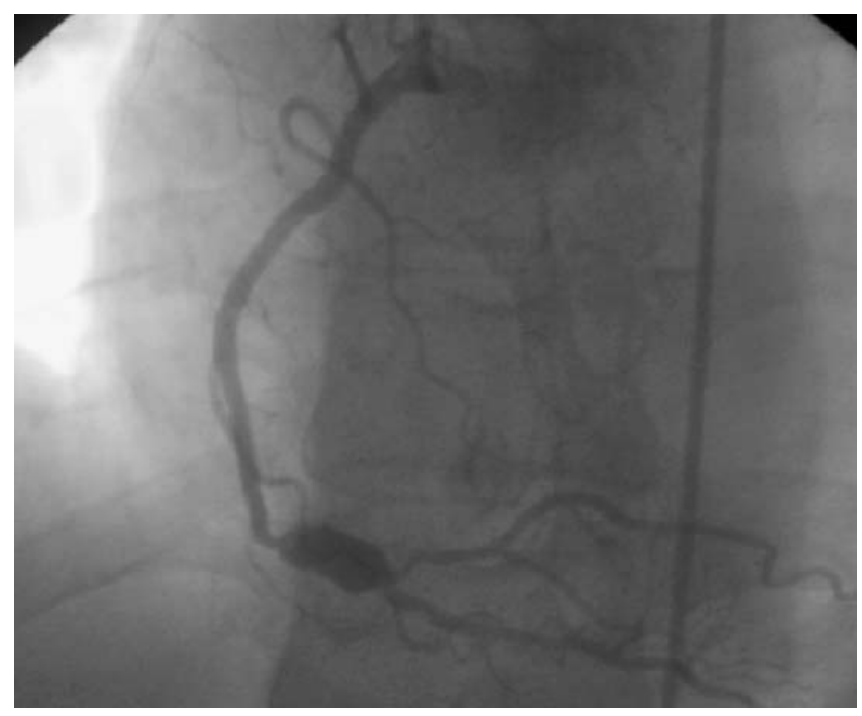

Fig. (1). Coronary angiography revealed an aneurysm of the right coronary artery with tight stenosis of the posterolateral branch. 
rosclerotic lesions and well developed collateral circulation. The electrocardiogram showed no signs of myocardial infarction and echocardiography also didn't show any abnormalities. Desobliteration of the right coronary artery was attempted but unsuccessful. Therefore, no coronary intervention was attempted and the current medical therapy was continued.

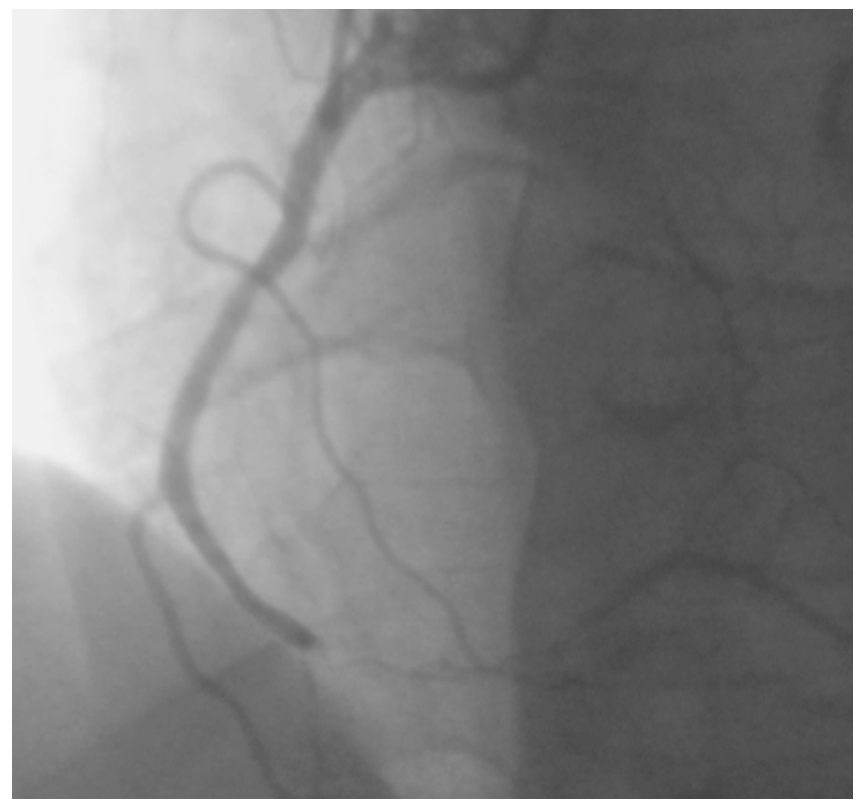

Fig. (2). Coronary angiography showed total occlusion of the right coronary.

\section{DISCUSSION}

$\mathrm{BD}$ is a chronic, multisystemic disorder characterized by inflammation of any size of the blood vessel wall. Vascular manifestations can be demonstrated in up to one third of the patients [2]. Venous manifestations are more common than arterial involvement [2]. Cardiac manifestations such as pericarditis, endomyocardial fibrosis, intracardiac thrombus, valvular disease occurred in about $5 \%$ of patients $[1,2]$. Although coronary artery disease is extremely uncommon, this complication posed several diagnostic and therapeutic challenges [5-9]. Coronary lesions can be either occlusive or aneurysmal degeneration, but coronary aneurysms are more frequent than stenotic lesions. The pathogenesis of the vasculitis is not well understood. The main targets are the endothelial cell with leucocytoclastic vasculitis resulting in obliterative endarteritis of the vasa vasorum and acute destruction of the vessel wall with formation of true or false aneurysms [2]. Arterial stenosis or occlusion is marked by adherent thrombus. The primary reason for clots seems to reside in the inflammatory process that leads to platelet aggregation and decrease in release of tissue plasminogen activator. The vascular thrombosis may be facilitated by trombophilic factor [1]. The case presented in this paper had coronary aneurysm and stenosis without myocardial infarction. The only vascular risk factors were moderate cigarette smoking and long term use of corticosteroid which are believed to have been accelerating factors for atherosclerosis. No coagulation and fibrinolytic system disorders were found. The coronary aneurysm and stenosis were managed with medical treatment with improvement of all symptoms, this evolution seemed to rule out atherosclerotic stenosis with dilatation. A follow-up angiogram revealed a total occlusion of the right coronary artery with atherosclerotic lesion. It was reported that BD such as chronic inflammation in lupus erythematosus lead to premature atherosclerosis, but Seyahi and al demonstrated recently that increased atherosclerotic is not prominent feature of BD. They suggest that coronary occlusion may be attributed to fibrous intimal thickening as a result of local vasculitis [5]. More than fourteen cases of coronary involvement associated with $\mathrm{BD}$ have been reported in the literature [6-14]. The affected patients are usually young male, only four women were reported $[4,8,11]$. Occlusion and obstruction were seen more frequently than aneurysm [4, 12]. Some previous reports showed that coronary arteritis may occur without myocardial infarction [4]. Similarly myocardial infarction can occur with a fully normal coronary angiogram. The authors suggesting coronary active vasculitis as a possible cause of acute myocardial infarction $[4,6]$.

Additional stenosis and aneurysm lesions of coronary artery are extremely rare and severe in BD. About 4 cases associated with BD have been reported in the literature [4, 12].

Coronary artery involvement is an uncommon phenomenon with controversial treatment strategy. According to our experience, medical treatment using immunosupressive agents must be started early [3]. On the other hand, considering the high mortality of myocardial infarction, it might be prudent to associate the standard therapy protocol. There is no consensus on the use of anticoagulants and thrombolytic agents but these drugs may cause fatal bleeding in the presence of aneurysms. For these reason, the medications must be used only in the absence of aneurysm and in association with an immunosupressant agent. Angioplasty and coronary stent implantation in occlusion coronary artery may cause rapid in-stent restenosis in $\mathrm{BD}$. Using covered stents for the aneurysm and surgical treatment is challenging because of the technical difficulties and postoperative complications, which include false aneurysm, anastomotic dehiscence and thrombotic occlusion $[14,15]$.

In conclusion, BD with coronary artery disease is very rare. Because of the risk of surgical intervention, immunosupressive therapy should be started with the standard therapy protocol of myocardial infarction with careful monitoring and follow-up of the patient.

\section{REFERENCES}

[1] Evereklioglu C. Current concepts in the etiology and treatment of Behçet disease. Surv Ophthalmol 2005; 50: 297-350.

[2] Wechsler B, Huong D L T, Kieffer E. Cardiovascular manifestations of Behçet's disease. Ann Med Interne 1999; 150: 542-54.

[3] Hammami S, Mahjoub S, Ben Hamda K, Brahem R, Gamra H, Ben Farhat M. Intracardiac thrombus in Behçet's disease : Two case reports. Thromb J 2005; 3: 9 (on line) Available from http://www. thrombosisjournal.com/qc/content/3/1/9

[4] Kraiem S, Fennira S, Battikh K, Chehaibi N, Hmem M, Slimane ML. Behçet disease : uncommon etiology of myocardial infarction. Ann Cardiol Angeiol 2004; 53: 109-13.

[5] Seyahi E, Ugurlu S, Cumali R, et al. Atherosclerosis in Behçet's syndrome. Semin Arthritis Rheum 2008; 38: 1-12.

[6] Kosar F, Sahin I, Gullu H, Cehreli S. Acute myocardial infarction with normal coronary arteries in a young man with the Behçet's disease. Int J Cardiol 2005; 99: 355-7.

[7] Arishiro K, Nariyama J, Hoshiga M, et al. Vascular Behçet's disease with coronary artery aneurysm. Intern Med 2006; 45: 903-7. 
[8] Tatli E, Surucu H, Aktoz M, Buyuklu M. Coronary artery ectasia in a patient with Behçet's disease. Saudi Med J 2007; 28: 1281-2.

[9] Yakut ZI, Odev K. pulmonary and cardiac involvement in Behçet disease : 3 case reports. Clin Appl Thromb Hemost 2007; 13: 31822.

[10] Cuisset T, Quilici J, Bonnet JL. Giant coronary artery aneurysm in Behçet's disease. Heart 2007; 93: 1375.

[11] Kwon CM, Lee SH, Kim JH, et al. A case of Behcet's disease with pericarditis, thrombotic thrombocytopenic purpura, deep vein thrombosis and coronary artery pseudo aneurysm. Korean J Intern Med 2006; 21: 50-6.
[12] Hattouri S, Kawana S. Behçet's syndrome associated with acute myocardial infarction. J Nippon Med Sch 2003; 70: 49-52.

[13] Geyik B, Ozdemir O, Ozeke O, Duru E. Giant left anterior descending artery aneurysm in a patient with Behcet's disease. Heart Lung Circ 2005; 14: 262.

[14] Sismanoglu M, Omeroglu SN, Mansuroglu D, et al. Coronary artery disease and coronary artery bypass grafting in Behcet's disease. J Card Surg. 2005: 20: 160-3.

[15] Kobayashi A, Sakata R, Kinjo T, Yotsumoto G, Matsumoto K, Iguro Y. Off-pump coronary artery bypass grafting in a patient with Behcet's disease. Jpn J Thorac Cardiovasc Surg 2004; 52: 527-9.

(C) Hammami et al.; Licensee Bentham Open.

This is an open access article licensed under the terms of the Creative Commons Attribution Non-Commercial License (http://creativecommons.org/licenses/by$\mathrm{nc} / 3.0 /$ ) which permits unrestricted, non-commercial use, distribution and reproduction in any medium, provided the work is properly cited. 